\title{
Three-Dimensional S-Matrix Simulation of Single-Electron Resonant Tunnelling Through Random Ionised Donor States
}

\author{
HIROSHI MIZUTA* \\ Central Research Laboratory, Hitachi, Ltd., Kokubunji, Tokyo 185, JAPAN
}

\begin{abstract}
This paper presents a numerical study of single-electron resonant tunnelling (RT) assisted by a few ionised donors in a laterally-confined resonant tunnelling diode (LCRTD). The 3D multi-mode S-matrix simulation is performed newly introducing the scattering potential of discrete impurities. With a few ionised donors being placed, the calculated energy-dependence of the total transmission rate shows new resonances which are donor-configuration dependent. Visualised electron probability density reveals that these resonances originate in RT via single-donor-induced localised states. The I-V characteristics show current steps of order $0.1 \mathrm{nA}$ per donor before the main current peak, which is quantitatively in good agreement with the experimental results.
\end{abstract}

Keywords: Resonant tunnelling, Lateral confinement, Ionised donor, Schrödinger equation, S-matrix, Fine structure

\section{INTRODUCTION}

LCRTDs exhibit an interesting interplay phenomenon of lateral quantization, Coulomb blockade, and impurity-induced tunnelling [1,2]. Recently a new inplane gated AlAs/GaAs/AlAs LCRTD[3] has been proposed to study the lateral confinement effects on RT. It has been shown that the size of the lateral confinement can be controlled by gate bias. Multiple current peaks have been observed in the valley current region of the small-area LCRTDs, and the peak splitting with increasing confinement has been shown to be consistent with the effects of lateral confinement.

An additional fine structure has been observed close to the current threshold of relatively large-area
LCRTDs [3]. The I-V characteristics for the devices show a series of plateau-like structures which are basically independent of the gate bias and obviously different from those due to the lateral confinement. Dellow et al. have pointed out RT through donor states for a possible cause [2]: an ionised donor in the quantum well will give a localised potential well and associated bound states through which electron tunnelling can occur for biases below the threshold voltage. The total number of donor sites in the active device region has been estimated from the background doping concentration of typically $10^{14} \mathrm{~cm}^{-3}$. This results in an estimate of 1-5 donors for the LCRTDs under consideration [3], which appears consistent with the proposed picture. The present work is

* Present address. Hitachi Cambridge Laboratory, Cavendish Laboratory, Madingley Road, Cambridge, UK, CB3 OHE. Tel: (01223) 467944. Fax: (01223) 467942. E-mail: mizuta@phy.cam.ac.uk 
intended to give a detailed numerical analysis of this phenomenon and to demonstrate the dependence of the I-V characteristics on the donor configuration in the device.

\section{THREE-DIMENSIONAL S-MATRIX SIMULATION}

The simulation technology used in this study is based on the multi-mode S-matrix simulation developed earlier [4] to investigate the lateral-mode non-conserving RT due to non-uniform lateral confinement in the conventional 0D-RTDs. The 3D time-independent Schrödinger equation is solved for the $3 \mathrm{D}$ potential distribution, $\mathrm{V}(\mathrm{x}, \mathrm{y}, \mathrm{z})$, (the $\mathrm{z}$-axis is chosen in the vertical direction) with the scattering boundary conditions. $\mathrm{V}(\mathrm{x}, \mathrm{y}, \mathrm{z})$ consists of the lateral confinement potential, $\mathrm{V}_{\mathrm{LC}}(\mathrm{x}, \mathrm{y}, \mathrm{z})$, the electron affinity, $\mathrm{V}_{0}(\mathrm{z})$, the potential along the channel due to an external bias, $\mathrm{V}_{\mathrm{EX}}(\mathrm{z})$, (both assumed to change only in the vertical direction), and the scattering potential due to ionised donors, $\mathrm{V}_{\mathrm{IM}}(\mathrm{x}, \mathrm{y}, \mathrm{z})$, :

$$
\mathrm{V}(\mathrm{x}, \mathrm{y}, \mathrm{z})=\mathrm{V}_{\mathrm{LC}}(\mathrm{x}, \mathrm{y}, \mathrm{z})+\mathrm{V}_{0}(\mathrm{z})+\mathrm{V}_{\mathrm{EX}}(\mathrm{z})+\mathrm{V}_{\mathrm{IM}}(\mathrm{x}, \mathrm{y}, \mathrm{z})
$$

In the present work $\mathrm{V}_{\mathrm{IM}}(\mathrm{x}, \mathrm{y}, \mathrm{z})$ is simply modelled by using a 3D delta function:

$$
\mathrm{V}_{\mathrm{IM}}(\overrightarrow{\mathrm{r}})=-\mathrm{V}_{0} \sum_{\left\{\overrightarrow{\mathrm{r}_{\mathrm{i}}}\right\}} \delta\left(\overrightarrow{\mathrm{r}}-\overrightarrow{\mathrm{r}_{\mathrm{i}}}\right)
$$

where $\left\{\vec{r}_{i}\right\}$ denotes the configuration of the ionised donors. In the following the coordinates of ionised donors are expressed by using lateral and vertical displacements from the centre of the device region, $\delta \mathrm{r}$ and $\delta \mathrm{z}$. The $3 \mathrm{D}$ scattering state, $\psi(\mathrm{x}, \mathrm{y}, \mathrm{z})$, is decomposed by using a complete set of $2 \mathrm{D}$ lateral eigenstates, $\varphi_{\gamma}(\mathrm{x}, \mathrm{y} \mid \mathrm{z})$, at every $\mathrm{z}$ point $(\gamma$ is a lateral mode index) which is obtained by solving the lateral $2 \mathrm{D}$ Schrödinger equation with the Dirichlet boundary conditions on the lateral surface. A 3D finite-difference lattice with typically $25 \times 25 \times 40$ nodes has been adopted to discretise the equation, which has a uniform spacing in the $\mathrm{x}$ and $\mathrm{y}$ dimensions and $\mathrm{a}$ non-uniform spacing in the $\mathrm{z}$ dimension. The lateral eigenenergies are first obtained up to a given value by using the bisection method following Householder's tridiagonalization. $\varphi_{\gamma}(\mathrm{x}, \mathrm{y} \mathrm{Iz})$ is then calculated by the inverse iteration method. As a result, the 3D Schrödinger equation is reduced to the following 1D scattering equation for the vertical components of the wavefunction, $\chi_{\gamma}(\mathrm{z})$, :

$$
\begin{array}{r}
\frac{\mathrm{d}^{2}}{\mathrm{dz} z^{2}} \chi_{\gamma}(\mathrm{z})+\mathrm{k}_{\gamma}^{2}(\mathrm{z}) \chi_{\gamma}(\mathrm{z})+\sum_{\gamma \prime}\left(2 \mathrm{C}_{\gamma, \gamma^{\prime}}^{(0,1)}(\mathrm{z}) \frac{\mathrm{d}}{\mathrm{dz}} \chi_{\gamma^{\prime}}(\mathrm{z})+\right. \\
\left.\mathrm{C}_{\gamma, \gamma^{\prime}}^{(0,2)}(\mathrm{z}) \chi_{\gamma^{\prime}}(\mathrm{z})-\frac{2 \mathrm{~m}^{*}}{\hbar^{2}} \mathrm{~V}_{\gamma, \gamma^{\prime}}^{\mathrm{IM}}(\mathrm{z}) \chi_{\gamma^{\prime}}(\mathrm{z})\right)=0
\end{array}
$$

where $\mathrm{k}_{\gamma}(\mathrm{z})$ denotes a complex wavenumber and $C_{\gamma, \gamma^{\prime}}^{(0,1)}, C_{\gamma, \gamma^{\prime}}^{(0,2)}$ the lateral-mode mixing coefficients [4]. Few-ionised-donor scattering coefficients, $V_{\gamma \gamma^{\prime}}^{\mathrm{IM}}(z)$, are defined by

$$
\mathrm{V}_{\gamma, \gamma^{\prime}}^{\mathrm{IM}}(\mathrm{z})=\int \mathrm{dx} \int \mathrm{dy} \varphi_{\gamma}^{*}(\mathrm{x}, \mathrm{y} \mid \mathrm{z}) \mathrm{V}_{\mathrm{IM}}(\mathrm{x}, \mathrm{y}, \mathrm{z}) \varphi_{\gamma^{\prime}}(\mathrm{x}, \mathrm{y} \mid \mathrm{z})
$$

These coefficients are calculated by making use of $\varphi_{\gamma}(\mathrm{x}, \mathrm{y} \mid \mathrm{z})$. The first derivative term of $\chi_{\gamma}(\mathrm{z})$ in Eq. (3) can be eliminated by applying the relevant unitary transformation [4] and so $\chi_{\gamma}(\mathrm{z})$ can be finally expressed as a superposition of plane waves with a set of renormalised complex wavenumbers which are obtained by solving the eigenvalue equation by the QL method. Thus the lateral-mode based scattering matrix (S-matrix) can be obtained from the continuity of probability flux. The total transmission rate is obtained by summing up the contributions from all the lateral-modes and finally the tunnelling current is calculated assuming the global coherent tunnelling.

\section{RESULTS AND DISCUSSIONS}

The numerical simulation has been performed for a relatively large area $\mathrm{AlAs}(4.2 \mathrm{~nm}) / \mathrm{GaAs}(5.6 \mathrm{~nm}) /$ $\operatorname{AlAs}(4.2 \mathrm{~nm}) \mathrm{LCRTD}$ in which the lateral eigenenergies are nearly degenerate and the contributions from different modes are hardly separable on the I-V curve. Because a virtually flat confinement is achieved in the fabricated LCRTDs [3], a lateral confinement is 


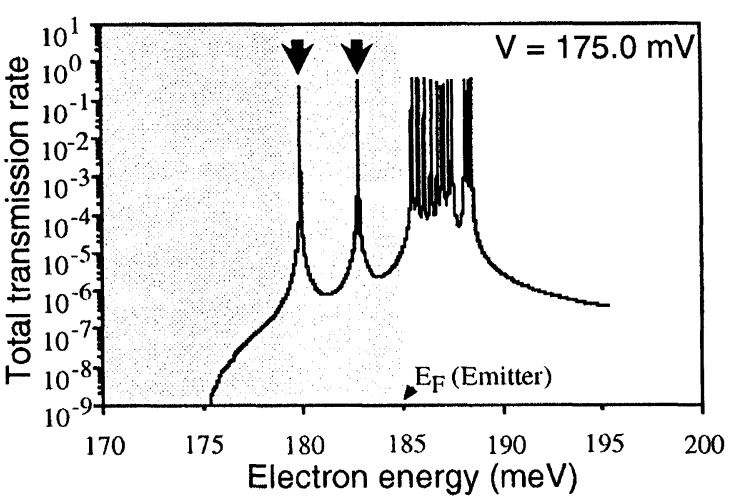

FIGURE 1 Energy dependence of total transmission rate calculated for a $200 \mathrm{~nm}$ diameter LCRTD in which two ionised donors are placed

assumed flat in the present simulation : a simple square potential well of $200 \mathrm{~nm}$ in diameter is used. Figure 1 shows the energy-dependence of the total transmission rate calculated for the LCRTD with two ionised donors being placed: one donor is at the centre, $(\delta \mathrm{r}, \delta \mathrm{z})=(0,0)$, and the other at $(\delta \mathrm{r}, \delta \mathrm{z})=(68 \mathrm{~nm}$, $1.3 \mathrm{~nm})$. In Fig. 1 two transmission peaks are seen at lower energiesapart from a series of peaks at higher

(a)

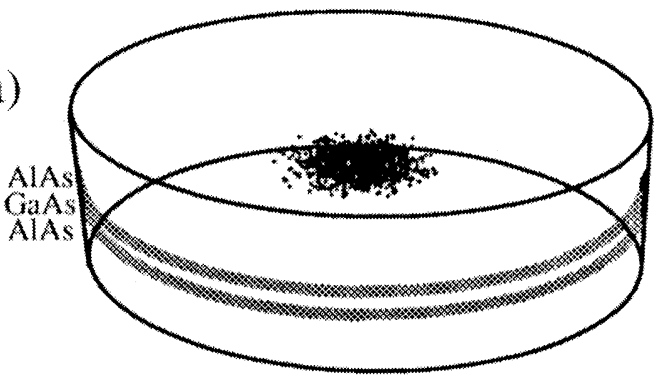

(b)

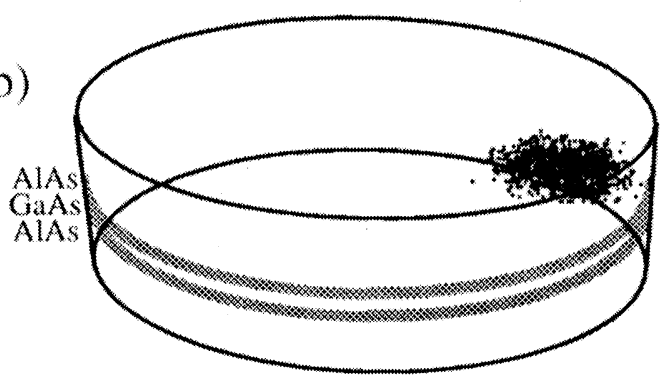

FIGURE 2 Visualised 3D electron probability density ,$|\psi(\mathrm{x}, \mathrm{y}, \mathrm{z})|^{* *} 2$, in the $200 \mathrm{~nm}$ diameter LCRTD with two ionised donors calculated for the 1 st mode incident wave from the emitter: (a) at first and (b) at second resonance energies. The latter higher energy peaks represent RT through the original quantised states whose energies are nearly degenerate since the size of the lateral confinement is fairly large. This simply gives rise to a large single negative differential conductance in the I-V characteristics (see Main peak in Fig. 3(a)) in the same way as conventional RTDs. On the other hands the new two peaks result from the ionised donors. The attractive potential of the ionised donors selectively pulls down some resonant states from the degenerate states. This is evident from the probability densities of electron calculated at these two resonant energies. Figure 2 shows the 3D visualisation of the probability density at (a) 1st and (b) 2nd resonances calculated for the 1st mode incident waves. It can be seen that the electron states are strongly localised at the different donor sites.

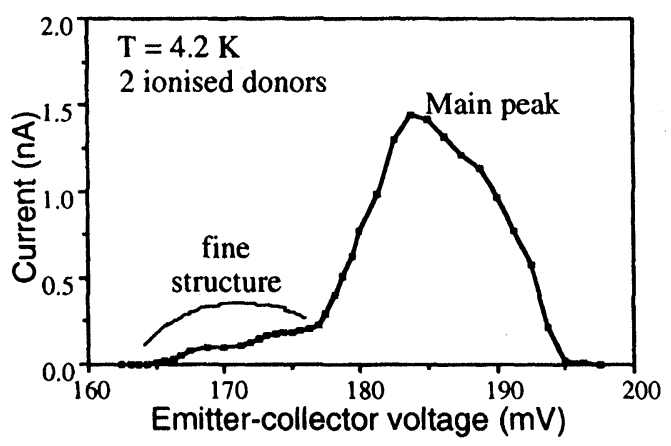

(a) Full I-V curve

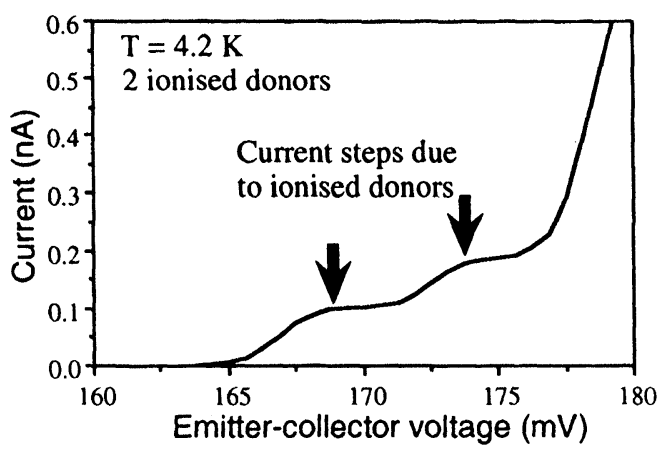

(b) I-V curve near current threshold

FIGURE 3 Simulated current-voltage characteristics for the $200 \mathrm{~nm}$ diameter LCRTD with two ionised donors : (a) for a whole voltage range and (b) near current thresold

The I-V characteristics calculated at $4.2 \mathrm{~K}$ are shown in Fig. 3 : (a) for an extended bias region and 
(b) near the current threshold. The ionised-donor-induced RT leads to double current plateaux with a step of about $0.1 \mathrm{nA}$ before the main current peak : this is in good agreement with the experimental results [3]. From this current the associated electron dwell time, $\tau_{\mathrm{d}}$, is estimated to be approximately $1500 \mathrm{ps}$ by the simple formula for single electron RT current, $\mathrm{I}=\mathrm{e} / \tau_{\mathrm{d}}$, [5]. This figure is close to the other experimental estimate of about 500 ps obtained by using the magnetoconductance measurements [5]. This fact also indicates that the observed current plateaux are attributable to RT mediated by ionised donors.

The fine structure of the I-V characteristics obviously depends on the configuration of the ionised donors. To manifest this the LCRTDs including only a single ionised donor has also been simulated. By changing the position of the single donor vertically and laterally in the active device region, it is found that the effect of the ionised donor is most enhanced when it locates at the centre of the device. By moving the donor from the centre to the edge of the device region, either horizontally or vertically, the single transmission peak due to the ionised donor gradually shifts to the higher energy and finally merges into the series of the peaks. In the I-V characteristics the subthreshold voltage of the single current plateau shifts towards the main peak voltage with keeping the current step virtually the same. This indicates that RT via a few ionised donors results in the complex current staircase depending on their configuration. Thus the calculated fine structures in general differ in the for- ward and reverse emitter-collector bias directions although the original double barrier structure is symmetrical. This is also consistent with the experimental results [3].

\section{Acknowledgements}

The author wishes to thank Prof. H. Ahmed and Dr. J. R. A. Cleaver of University of Cambridge, and Dr. K. Nakazato of Hitachi Cambridge Laboratory for their valuable discussions.

\section{References}

[1] Bo Su, V. J. Goldman, and J. E. Cunningham, "Single-electron tunneling in nanometer-scale double-barrier heterostructure devices", Phys. Rev. B46, 7644, 1992.

[2] M. W. Dellow, P. H. Beton, C. J. G. M. Langerak, T. J. Foster, P. C. Main, L. Eaves, M. Henini, S. P. Beaumont and C. D. W. Wilkinson, "Resonant tunneling through the bound states of a single donor atom in a quantum well", Phys. Rev. Lett. 68, 1754, 1992.

[3] C. J. Goodings, H. Mizuta, J. R. A. Cleaver, and H. Ahmed, "Variable-area resonant tunnelling diodes using implanted in-plane gates", J. Appl. Phys.76, 1276, 1994.

[4] H. Mizuta, C. J. Goodings, M. Wagner, and S. Ho, "Three-dimensional numerical analysis of multi-mode quantum transport in zero-dimensional resonant tunnelling diodes", J. Phys.: Condens. Matter 4, 8783, 1992.

[5] H. Mizuta and T. Tomonori, 1995, The Physics and Application of Resonant Tunnelling Diodes (Cambridge University Press), Chapters 4 and 6.

\section{Biography}

Hiroshi Mizuta has served as a researcher at the Central Research Laboratory, Hitachi Ltd., and Hitachi Cambridge Laboratory, Hitachi Europe Ltd. His current research interest includes numerical simulation of heterojunction electron devices and quantum transport modeling. 

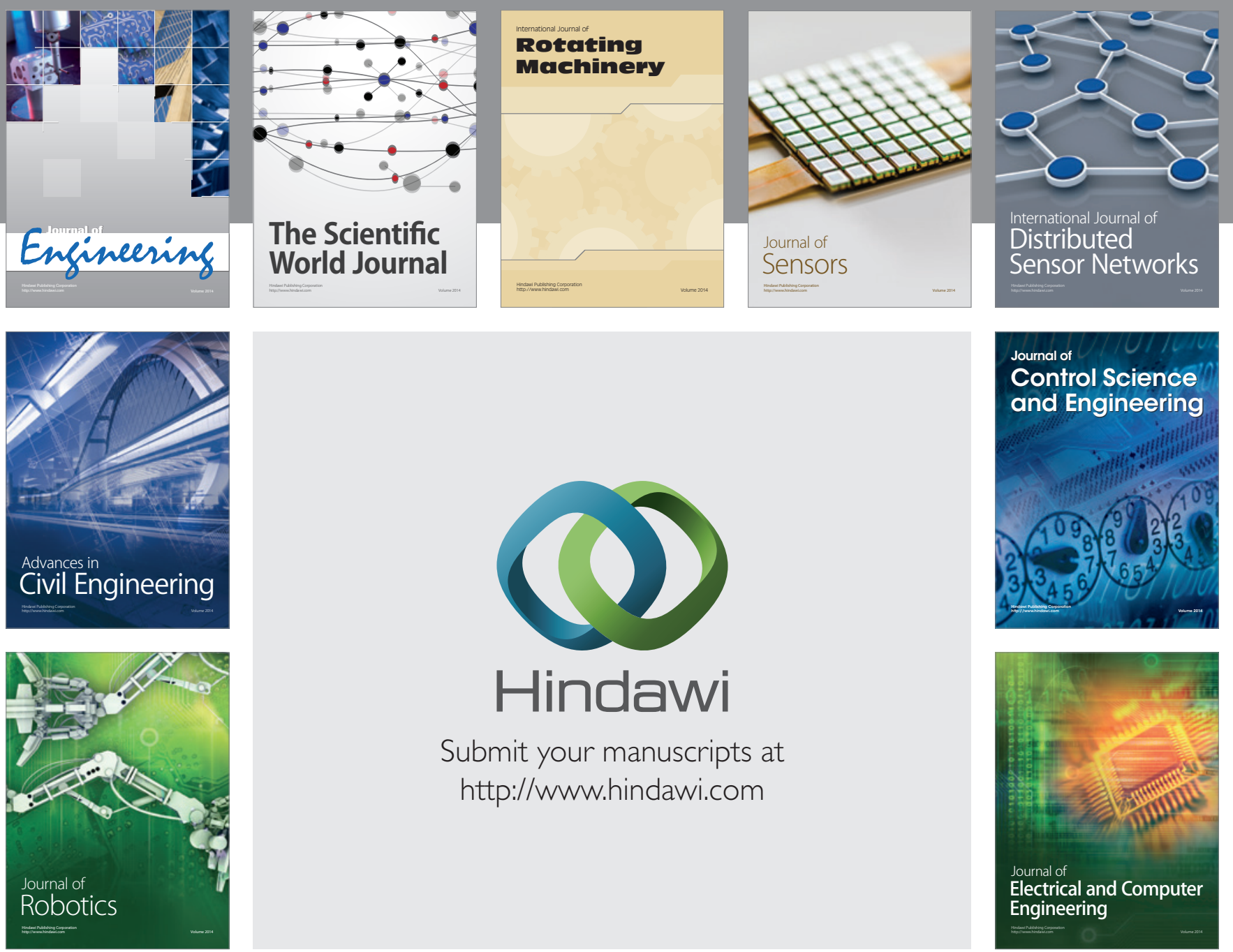

Submit your manuscripts at

http://www.hindawi.com
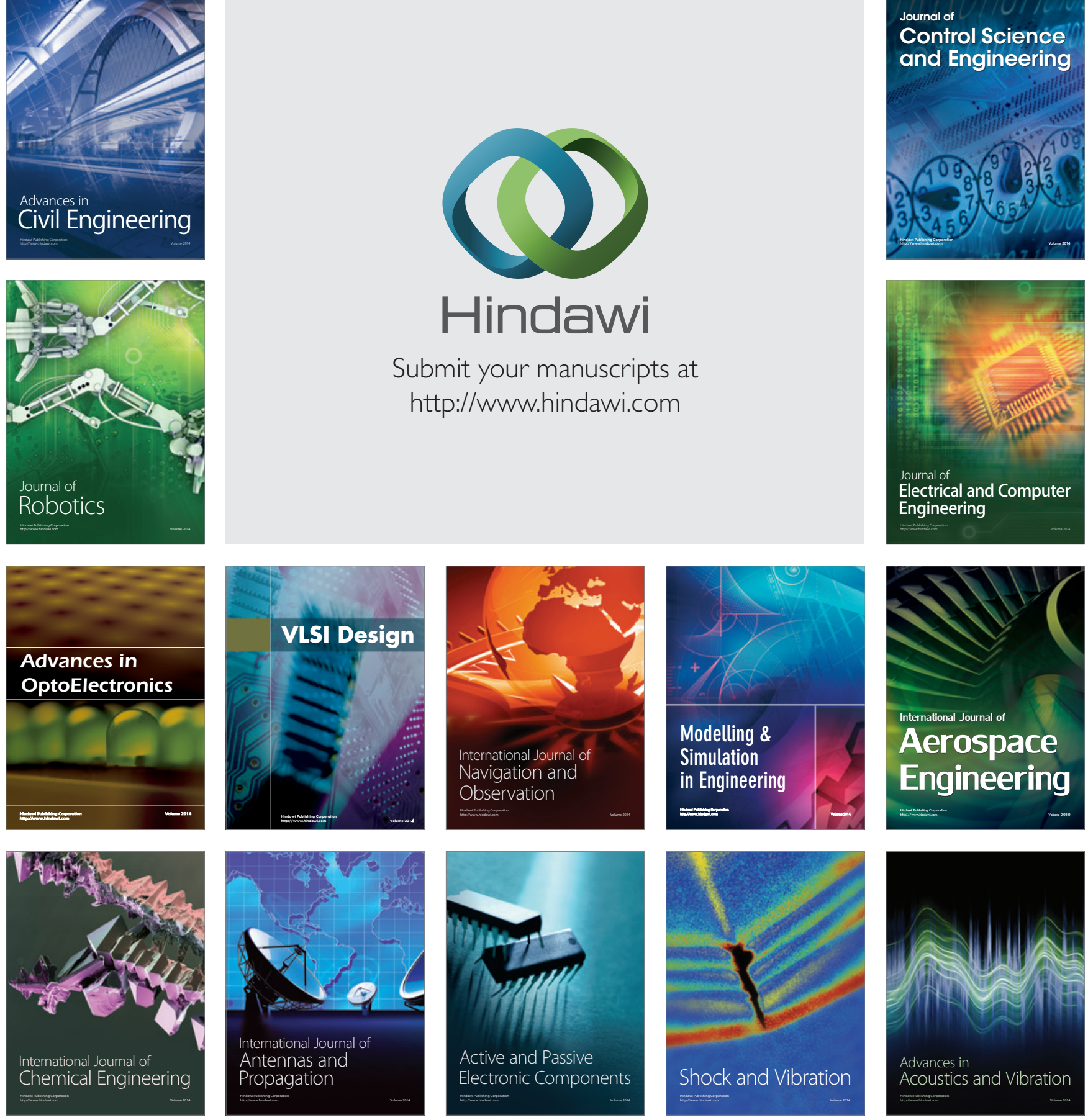